Europäisches Kompetenzzentrum für Asthma und Allergien

Die Deutsche Hochgebirgsklinik Davos- Wolfgang, die Allergieklinik Davos (Zentrum für Kinder und Jugendliche) und das Niederländische Asthmazentrum-Davos haben sich zu einer bisher einzigartigen Kooperation zusammengefunden und im April diesen Jahres das Europäische Allergie- und Asthma-Centrum Davos (EACD) aus der Taufe gehoben. Das neue internationale Kompetenzzentrum ist spezialisiert auf die Behandlung von Patienten mit Asthma bronchiale, COPD mit relevanter bronchialer Hyperreagibilität und komplexen Multiorgan-Allergien.

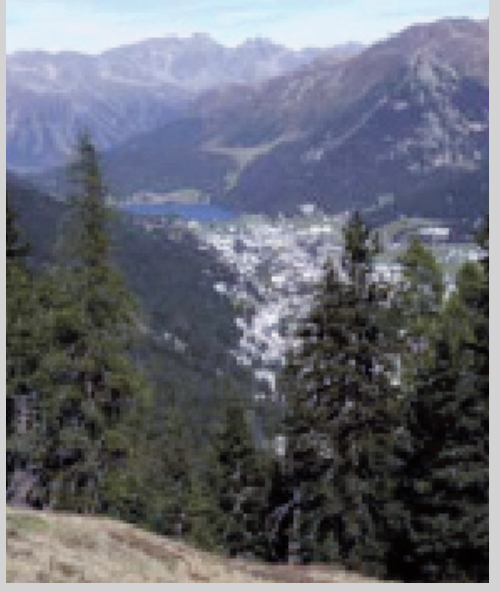

Die Lage des EACD im Davoser Hochtal, das im Mittel 1.60o Meter über dem Meer liegt, ist geradezu prädestiniert zur pneumologischen Rehabilitation. Die Belastung durch Luftschadstoffe wie Stickoxide, Schwefeldioxid, Ozon und Staub ist im Vergleich zum Flachland viel geringer. Auch die durch Schadstoffe kaum belasteten Pollen weisen deutlich weniger aggressive Allergene auf, und schließlich fühlen sich auch Hausstaubmilben in dieser Höhe nicht mehr wohl.

Das EACD engagiert sich über die therapeutische Betreuung hinaus auch in der Grundlagenforschung, die in Kooperation mit dem Schweizer Institut für Allergie- und Asthma-Forschung (SIAF) durchgeführt wird.

Weitere Informationen bietet unter www.eacd.net die Homepage des Zentrums.

\title{
Kooperationspflicht gefordert
}

\section{Der 107. Deutsche Ärztetag}

im Mai in Bremen hat das

Gesundheitsministerium aufgefordert, Pharmafirmen gesetzlich dazu zu verpflichten, die Einzelbestandteile ihrer Präparate zur Abklärung von Überempfindlichkeitsreaktionen herauszugeben.

Z ur allergologischen Diagnostik von Überempfindlichkeitsreaktionen auf Arzneipräparate werden die Inhaltsstoffe der verdächtigen Zubereitungen für die erforderlichen Tests (Hauttests, In-vitro-Tests, Provokationstests) benötigt. Meist werden diese von den pharmazeutischen Unternehmen zur Verfügung gestellt. Um dies nun in allen Fällen sicherzustellen, hat der 107. Deutsche Ärztetag den Gesetzgeber aufgefordert, diesen Beitrag der pharmazeutischen Unternehmen zur Arzneisicherheit verpflichtend zu machen. Es besteht die Hoffnung, dass Überempfindlichkeitsreaktionen auf Arzneimittel in Zukunft von den behandelnden Allergologen besser und in guter Zusammenarbeit mit den pharmazeutischen Unternehmen geklärt werden können.

Dr. Claudia Borelli, München

\section{Entschließung des \\ 107. Deutschen Ärztetages}

Das Gesundheitsministerium wird auf gefordert, im Arzneimittelgesetz eine gesetzliche Grundlage zu schaffen, die deutsche und ausländische Pharmafirmen, die Präparate in Deutschland anbieten, verpflichtet, die Einzelbestandteile der Präparate zur Abklärung allergischer Reaktionen an anfordernde Ärzte herauszugeben.

\section{Begründung}

Pharmafirmen sind derzeitig nicht gesetzlich verpflichtet, die einzelnen Bestandteile ihrer Arzneistoffe an Ärzte weiterzugeben, die diese Einzelbestandteile zur Klärung allergischer oder gar lebensbedrohlicher anaphylaktischer Reaktionen auf die jeweiligen Präparate zur Testung ihrer Patienten benötigen. Bei Nichtherausgabe der Einzelbestandteile durch die Pharmafirmen, die immer wieder vorkommt, bleiben wichtige allergische Reaktionen oder gar lebensbedrohende anaphylaktische Reaktionen der Patienten unabgeklärt. Den Patienten kann ärztlicherseits nicht geraten werden, welche Medikamente vertragen werden und welche Stoffe oder Bestandteile der Medikamente gemieden werden müssen.

Beschluss des 107. Deutschen Ärztetages. Dt Ärzteblatt 2004; 101: B 1327

\section{Preis für Münchener Pädiater}

\section{Kinder mit einem Defekt an den Genen GSTM1 und GSTT1 haben ein} erhöhtes Risiko, aufgrund von Passivrauchen Asthma zu entwickeln. Für diese Erkenntnis erhielt Dr. Michael Kabesch anlässlich der 26. Jahrestagung der Deutschen Gesellschaft für Pädiatrische Pneumologie in Hamburg den „Klosterfrau-Forschungspreis 2004“.

G STM1 und GSTT1 sind Gene, die bei der Entgiftung von Tabakrauch im menschlichen Körper eine zentrale Rolle spielen. Ziel der Prävalenzstudie von Kabesch war es, den Einfluss eines genetisch bedingten Mangels an GST-Enzymen in Verbindung mit Passivrauchen in Hinblick auf Entwick- lung und Schwere atopischer Erkrankungen zu untersuchen. Dazu wurden Eltern anhand eines Fragebogens zur Gesundheit der Atemwege ihrer Kinder sowie über die eigenen Rauchgewohnheiten befragt. Bei den Kindern wurden ein Pricktest durchgeführt sowie die Lungenfunktion und bronchiale Reiz- 